\title{
LES CRISTAUX LIQUIDES FERROÉLECTRIQUES ET LEUR UTILISATION EN OPTOELECTRONIQUE
}

\author{
J. P. LE PESANT \\ THOMSON-CSF, Laborato1re Central de Recherches, Domaine de \\ Corbeville, F-91404 orsay Cedex, France
}

\section{RESUEF}

La découverte de la ferroélectricité dans les cristaux liquides smectiques C chiraux, en 1975, a conduit à un nouvel effet electrooptique induit par un couplage linéaire entre la polarisation électrique permanente des molécules et le champ électrique $d^{\prime}$ adressage. Les cristaux liquides ferroélectriques permettent une réduction $d^{\prime}$ au moins 3 ordres de grandeur du temps de commutation par rapport aux nématiques en hélice traditionnels, et permettent $1^{\prime}$ obtention d'états stables permanents utilisés pour des effets à mémoire (stabilisation par effet de surface pour les cellules de faible épaisseur et stabilisation en courant alternatif pour les cellules de faible et moyenne épaisseur).

Tout ceci a ouvert la voie a toute une gamme de dispositifs nouveaux et plus rapides qui $s^{\prime}$ étend des écrans de visualisation fortement multiplexés jusqu'aux obturateurs optiques.

Après quelques rappels sur les structures de phases des cristaux liquides, nous présentons les caractères spécifiques des cristaux liquides ferroélectriques.Nous exposons ensuite les proprietés de bistabilité par effet de surface et par maintien en champ électrique alternatif. Nous aborderons 1'aspect matériaux par la description des tendances actuelles dans la synthèse des matériaux et $1^{\prime}$ ancrage moléculaíre.Nous donnerons quelques éléments sur la technologie de fabrication des cellules. Après un bref exposé sur les écrans de visualisation à cristaux liquides ferrólectriques, nous décrirons plusieurs dispositifs électooptiques rapides tels qu'obturateurs et modulateurs optiques.Nous verrons que des dimensions de points de 1'ordre de la dizaine de micrometres et des temps de commutation inférieurs a 20 microsecondes sont maintenant obtenus dans les laboratoires.De nombreuses références bibliographiques sont données en annexe.

\section{ABSTACT}

The discovery of ferroelectricity in smectic C liquid crystals, in 1975, has introduced a new electrooptic effect, driven by linear coupling between the permanent electric polarization of chiral smectic C molecules and the addressing electric field. Ferroelectric liquid crystals allow a reduction of at least 3 orders of magnitude of the switching time with respect to conventional twisted nematics and yield permanent stable states used for memory effects (surface stabilization for small thickness cells and AC stabilization for small and medium thickness cells). All this has open the way to new and faster devices ranging from highly multiplexed displays to optical shutters. This paper describes the present trends in material synthesis, molecular anchoring and device fabrication. New mixtures have been prepared in several laboratories, which yield low viscosity materials by using high polarity chiral molecules as dopants. Response times of less than 40 us are currently obtained and lower ones are forseable. Small pitch (less than $50 \mathrm{um}$ ) optical shutters are described and applications such like line printers, light modulators and optical gate arrays will be dicussed. 


\section{INTRODUCTION}

Les cristaux liquides ont maintenant à peine plus de cent ans, puisque $c^{\prime}$ est en 1988 que Reinitzer découvrit des phases de matière condensée qui présentent des propriétés physiques intermédiaires entre celles des solides et celles des liquides.Elles ont été appelées depuis mésophases ou plus communément "cristaux liquides". Les physiciens les ont reconnues comme de véritables états thermodynamiques et ont étudié en détail leur structure et leur comportement aussi bien du point de vue théorique qu'expérimental. L'une de ces phases, la phase nématique, qui est obtenue avec de nombreux matériaux, pures ou en mélanges, est maintenant largement utilisée dans l'état dit "nématique en hélice", dans les montres, les calculatrices de poche, les microordinateurs portables, les télévisions à cristaux liquides etc... D'autres phases, en particulier les phases smectiques, permettent des temps de réponse plus courts, des angles de vue très larges, des dimensions de points très faibles et des états mémoires intrinsèques ou entrete nus. Le but de ce cours était de décrire quelques uns des progrès qui ont été faits dans la mastrise des matériaux, de l'orientation moléculaire, de la technologie des cell ules et des effets électrooptiques. Ces progrès permettent maintenant la fabrication d'obturateurs optiques rapides et compacts utilisables dans des applications telles que traitement de faisceau, modulateurs optiques et barrettes pour imprimantes électrooptiques.

\section{STRUCTURE ET SMETRIE DES CRISTAUX LIQUIDES}

De nombreuses molécules organiques présentent des phases cristal liquide, soit elles-mêmes à l'état pur, soit à $1^{\prime}$ état de mélange avec $d^{\prime}$ autres molécules qui ne présentent pas intrinsèquement $1^{\prime}$ état mésomorphe. Deux grandes classes sont actuellement distinguées: les lyotropes et les thermotropes.

Les lyotropes sont composés de molécules amphiphiles; leur comportement et leur structure de phase sont contrôlés par la température et la concentration relative des constituants non miscibles. Actuellement il $n^{\prime} y$ a pas eu de développement. $d^{\prime}$ applications pratiques de ces phases aux dispositifs électrooptiques.

Les thermotropes sont des matériaux purs ou des mélanges moléculaires, dont les molécules ne sont pas nécessairement amphiphiles, et dont le comportement est contrôlé par la température. C'est uniquement cette classe des thermotropes, et parmi eux uniquement les molécules de faible poids moléculaire, que nous considèrerons ici. On pourra trouver ailleurs [1] des références concernant les molécules de poids moléculaire plus élevé (polymères cristaux liquides).

La plupart des thermotropes de faible poids moleculaire ont des molécules allongées en forme de bâtonnet comportant un corps mésogène (aromatique ou alicyclique) et des groupes terminaux attachés à l'une ou l'autre ou aux deux extrémités.

Deux grandes classes de structures sont utilisées pour décrire le polymorphisme des critaux liquides (CL): les nématiques et les smectiques (Fig.1).

Dans les nématiques, les molécules sont distribuées au hasard mais leur axe d'élongation pointe localement approximativement dans une même direction appelée le directeur $\mathrm{n}$. Lorsque des conditions aux limites approprikes et suffisamment fortes sont imposées, cette direction est la même sur de longues distances en comparaison des dimensions moléculaires. La structure la plus largement utilisée actuellement est le nématique en hélice ("twisted nematic" T.N.): les molécules sont ancrées avec une faible inclinaison sur chaque face de la cellule à CL dans des directions qui sont respectivement perpendiculaires 1'une à 1'autre ; ceci conduit, pour des matériaux et des épaisseurs appropriés, à un quart du pas d'une structure hélicoidale qui agit en guide d'onde pour la lumière polarisée, pour des longueurs $d^{\prime}$ onde qui satisfont la condition de Mauquin. On trouvera en Annexe I les équations de couplage avec le champ électrique.

Les temps de commutation typiques pour de faibles tensions appliquées sont de quelques dizaines de millisecondes pour 2 à 20 volts sur des cellules de 5 à 50 um d'épaisseur.Il $n^{\prime} y$ a pas d'effet mémoire intrinsèque mais l'état comnuté peut 
être maitenu localement sur les pixels par des moyens extérieurs, par exemple des transistors en couche mince ("thin film transistors" TFT) [2]. Des temps de réponse plus courts ont été publiés pour des matériaux de haute pureté sous fort champ électrique [3]. Si une phase nématique est constituée de molécules chirales, une stucture torsadée, appelée cholestérique $\left(\mathbb{N}^{\star}\right)$, se superpose à la structure nématique, avec un axe d'enroulement perpendiculaire au directeur $n$. Le pas de cette torsade est fortement dépendant de la température et de la pression $d^{\prime}$ où une dépendance selon ces paramètres de l'aspect coloré dú aux réflexions de Bragg sur la stucture hélicoidale périodique.

Dans les smectiques, les molécules sont davantage ordonnées: leurs centres de gravité sont dans des plans parallèles les uns aux autres.

Deux sous-classes ont été largement étudiées:

- les smectiques A dans lesquels les molécules ont leur grand axe perpendiculaire aux plans;

- les smectiques C dans lesquels le grand axe est incliné par rapport aux plans, de telle sorte que les molécules peuvent tourner sur un cône d'axe perpendiculaire aux plans.

La structure smectique est plus proche de celle du cristal que celle des nématiques et conduit dans certains cas à des temps de réponse plus courts (1 à $500 \mu \mathrm{s})$ et à des effets de mémoire intrinsèques. Si les molécules sont chirales,les syméties de la structure sont modifiées. Dans le cas général, une stucture torsadée se superpose à la structure smectique, avec un axe hélicoïdal perpendiculaire aux plans smectiques. Les conséquences de ces structures sont discutés ci-dessous.

\section{FERROBLECTRICITE DANS LES CRISTAUX LIQUIDES.}

La ferroélectricité dans les cristaux liquides à été prévue et démontrée en 1975 par Meyer et al. [4] en se basant sur les arguments suivants: dans les phases smectiques C ordinaires, la symétrie est monoclinique et le groupe de symétrie contient seulement un axe de rotation d'ordre deux parallèle aux couches et normal au grand axe moléculaire, un plan de symétrie perpendiculaire à l'axe de rotation, et un centre $d^{\prime}$ inversion. Cependant, si les molécules sont chirales (non superposables à leur image dans un miroir), le plan de symétrie et le centre $d^{\prime}$ inversion sont supprimés. L'axe de rotation d'ordre deux permet l'existence d'un moment dipolaire permanent parallèle à cet axe.

Ceci ouvre la voie à un couplage linéaire entre ce moment dipolaire et un champ électrique appliqué, qui constitue la différence fondamentale par rapport au couplage diélectrique, quadratique en champ, utilisé dans les nématiques, et qui cst lić au dipôle induit par le champ électrique lui même (Annexe I).

Les premières mesures furent faites sur le composé DOBAMBC à des températures comprises entre 70 et $90^{\circ} \mathrm{C}$. Actuellement, les progrès faits dans la synthèse des matériaux ont conduit à des phases smectiques C chirales (SmC ${ }^{\star}$ ) à température ambiante.

Comme nous l'avons dit plus haut, 1'observation de la ferroélectricté macroscopique nécessite le déroulement de la structure hélicoidale. Une expression générale donnant le champ critique de déroulement est donnée dans la référence [4]. Des expressions plus détaillées ont été obtenues [5] pour diverses orientations moléculaires.

Le déroulement de la structure hélicoidale peut être obtenu sur de très grandes surfaces et il est plus aisé pour des matériaux $\mathrm{CL}$ de grand pas et pour certaines séquences $\mathrm{CL}$ en température. Le couplage diélectrique entre un champ alternatif et le dipôle induit, qui existe aussi, peut aider à dérouler l'hélice. Ceci est même encore plus efficace, comme nous le verrons plus tard lorsque les molécules tendent à s'aligner perpendiculairement au champ alternatif appliqué, $c^{\prime}$ est à dire lorsqu'elles possèdent une anisotropie diélectrique négative.

La réorientation des molécules sous des champs électriques continus ou alternatifs dépend partiellement de la viscosité de rotation. Ainsi,bien qu' on puisse espérer que les temps de réponse les plus courts soient associés aux polarisations les plus élevées, dans l'état actuel des connaissances [6], les composés qui 
ont les plus fortes polarisations ont aussi les plus fortes viscosités de rotation. Actuellement, cette difficulté est contournée par la préparation de mélanges dopés dans lesquels des molécules de forte polarisation sont dissoutes dans des solvants de faible viscosité. Ceci montre 1'importance cruciale du génie chimique pour les cristaux liquides ferroélectriques.

\section{STROCTURE MOLRCULAIRE DES CRISTAUX LIQUIDES FRRROBLECTRIQUES}

Depuis 1976, plusieurs centaines de molécules de SmC^ ont été synthétisées. 1.a structure générale de ces composés est reproduite en Annexe II, ainsi que quelques exemples de molécules qui donnent de fortes polarisations et des temps de réponse très courts.La structure générale est composée de deux ou trois cycles carbonés et de chaines terminales portant un ou plusieurs carbones asymétriques.

Des travaux récents [36] ont jeté les bases d'un modèle pour 1'origine moléculaire de la polarisation des CL ferroelectriques. Les auteurs montrent en particulier l'importance des configurations multiples que peuvent prendre les groupes terminaux, très flexibles, par rapport au coeur plus rigide de la molécule. Ces groupes sont beaucoup plus désordonnés("fondus") que le coeur et donc occupent beaucoup plus de volume que lui. Ceci conduit, pour rendre l'énergie minimum, à une configuration des SmC* dans laquelle les groupes terminaux sont moins inclinés par rapport aux couches que les coeurs des molécules.Des études par rayons $X$ à haute résolution [37] sur un phenyle benzoate apportent une confirmation expérimentale de ces hypothèses.

Nous reproduisons en Annexe III quelques figures extraites de cette référence, que nous avons utilisées dans l'exposé oral.

\section{BISTABILITE: STABILISATION EN SURFACE ET EN CHAMP ALTERNATIF}

Nous avons vu plus haut qu'il est nécessaire de dérouler la structure hélicoïdale pour obtenir une polarisation macroscopique. Il faut donc maintenir ce déroulement de l'hélice pour rendre visible l'effet électrooptique. L'application d'un champ électrique est couramment utilisée pour dérouler l'hellice, mais lorsque le pas de celle ci est supérieur à l'épaisseur de la cellule, un nettoyage approprié et un traitement des surfaces de confinement conduisent aussi à ce déroulement. Cependant, ce déroulement n'est maintenu que pour les faibles épaisseurs de cellule (1 à $2 \mu \mathrm{m})$; on obtient alors la stabilisation de surface (SSFLC:surface stabilized ferroelectric liquid crystal). Lorsque les épaisseurs sont supérieures ou égales à 3 ou $4 \mu \mathrm{m}, 1^{\prime}$ orientation des molécules $\mathrm{n}^{\prime}$ est pas maintenue quand le champ d'adressage est supprimé, ce qui produit des domaines d'orientations diverses.

En 1980, Clark et Lagerwall ont découvert et breveté que, pour des épaisseurs de cellules inférieures à 2 m et une géométrie moléculaire telle que les couches smectiques soient perpendiculaires aux surfaces de confinement ("book shelf geometry"), on obtient la bistabilité [17]. Dans ce cas, les molécules restent dans la position où les a amenées la force de couplage linéaire avec le champ électrique. Avec des conditions aux limites appropriées sur les surfaces de confinement, on peut avoir des molécules quasi-parallèles à ces surfaces dans $1^{\prime}$ un ou l'autre des états d'orientation de la polarisation ("up" ou "down"). On trouvera unereprésentation schématique sur la figure 2.

Un autre moyen d'obtenir la bistabilité repose sur le couplage quadratique entre le champ électrique appliqué $\mathrm{E}$ et le dipôle induit, qui produit un couple de rotation proportionnel à l'anisotropie diélectrique. Lorsque cette anisotropie est négative, le champ appliqué tend à orienter les molécules perpendiculairement a la direction du champ électrique $E$, quelle que soit sa polarité, puisque 1'interaction est quadratique. Ceci a été breveté et publié par le pesant et al. en 1984 [18] et constitue une mémoire entretenue qui permet, d'une part d'améliorer la qualité d'orientation et de mémoire dans les cellules de faible épaisseur, et d'autre part d'utiliser dans certains cas des cellules de plus forte épais- 
seur ( 4 à $8 \mu \mathrm{m}$ typiquement). Ceci constitue la stabilisation en champ alternatif(ACS FLC: AC stabilized ferroelectric liquid crystal).

Actuellement, les deux effets sont utilisés conjointement pour obtenir une bonne bistabilité et des taux de multiplexage élevés, et pour rendre minimum les interactions entre pixels.

\section{TECHNOLOGIE DES CELLULBS}

$\mathrm{L}^{\prime}$ ancrage et $\mathrm{l}^{\prime}$ orientation des molécules de cristal liquide nématique sur de grandes surfaces est de nos jours bien maltrisé. Pour les applications commerciales, on utilise des polymères frottés (par exemple des polyimides) comme couche $d^{\prime}$ alignement. La phase smectique chirale ferroélectrique est plus difficile à orienter parcequ'elle est plus visqueuse et qu'il faut contôler à la fois l'orientation des couches et celle du directeur moléculaire.

Certains auteurs font état de l'utilisation de polyimide frotté pour la stabilisation en alternatif $[18,19]$ mais d'autres polymères ont été essayés depuis et des résultats expérimentaux d'essais systématiques sur différentes familles de cristaux liquides ont été publiés [20]. A Thomson-CSF, les meilleurs résultats ont été obtenus avec des frottements parallèles ou anti-parallèles des substrats traités soit avec du polyvinyl alcool (épaisseur $100 \mathrm{~nm}$ ) soit avec du Nylon $6 / 6$ (épaisseur $30 \mathrm{~nm}$ ). Les défauts en "zig-zag" ont été fortement réduits et même éliminés pour certains des composés. Cependant, un travail important reste à faire sur les matériaux cristaux liquides et 1'ancrage car actuellement, les échantillons les mieux orientés ne sont pas nécessairement ceux qui présentent la meilleure bistabilité.

Le choix des cales d'épaisseur est crucial pour le contrôle de l'uniformité de l'épaisseur des cellules. Plusieurs laboratoires ont utilisé des fibres de verre attaquées à l'acide fluorhydrique et calibrées au diamètre désiré. Ceci conduit à des difficultés technologiques quand 1'épaisseur demandée est seulement de quelques micromètres. Des sphères de polystyrène peuvent aussi être utilisées, en particulier en dispersion dans la couche $\mathrm{d}^{\prime}$ ancrage et $\mathrm{d}^{\prime}$ orientation, avant son dépôt, mais il faut alors empêcher 1'aggrégation des sphères pour éviter des surépaisseurs locales. Des cales d'épaisseur formées par un procédé de masquage ont $1^{\prime}$ avantage de permettre un positionnement précis de ces cales d'épaisseur entre les pixels, en dehors des zones utiles du dispositif. Des réalisations selon ce type de technologie ont été obtenues d'une part à l'Université de Boulder (Colorado) avec des cale d'épaisseur en monoxyde de silicium [21] et $d$ 'autre part à CEA-D.LETI [22] et à Thomson-CSF/LCR avec des cales en résine photoresist.

Le remplissage des cellules est une autre opération critique pour la fabrication des dispositifs à SmC*, à cause de la viscosité qui oblige à chauffer lors du remplissage et donc à tenir compte de l'existence de séquences de phases entre le Sm $\mathrm{C}^{\star}$ et le liquide isotrope. Actuellement, les meilleurs résultats sont obtenus en remplissant les cellules dans la phase lquide isotrope et en les laissant refroidir lentement. Un traitement thermique final sous champ électrique alternatif permet l'élimination de la plupart des défauts en zig-zag. Les structures et les défauts dans les smectiques chiraux ont été étudiés en particulier par M. Brunet [23] et divers auteurs cités dans cette référence. Avec les matériaux actuellement disponibles, une séquence comportant les phases cholestérique et smectique $A\left(I-N^{*}-S m A-S m C^{\star}\right)$ semble étre la plus appropriée. Une étude par diffusion des rayons $X$ à haute résolution de la structure locale en "chevron" des couches SmC* stabilisées en surface a été faite par Riecker et al. [24] en fonction de la température; elle montre l'influence de la présence initiale de défauts dans la phase SmA. 


\section{DISPOSITIFS DE VISUALISATION}

De nombreux travaux de recherche portent actuellement sur la fabrication de dispositifs de visualisation a cristaux liquides ferroélectriques, qui ont l'avantage de la rapidité(quelques dizaines de microsecondes par ligne), de la mémoire intrinsèque (SSFLC) ou entretenue (ACS FLC) et $d^{\prime}$ un très large angle de vue. Les premiers prototypes de grandes dimensions ont été présentés avec des images fixes dès 1985 par Seiko Instruments au Japan Electronic Show à Osaka.Des progrès ont été faits depuis pour obtenir des images vidéo et bientôt des images couleur avec teintes de gris [22-26]. Un écran en noir et blanc de 6 pouces de diagonale avec 9 teintes de gris a été présenté par CEA-D.LETI à IDRC 1988 à San Diego et Canon met au point un écran de format A4 compatible avec la haute qualité bureautique (SID 1989).

\section{OBTURATBURS}

Les propriétés spécifiques des cristaux liquides ferroélectriques, rapidité et bistabilité, les rendent très attractifs pour la réalisation d'obturateurs optiques compacts, de forte densité et potentiellement de faible cout. Nous donnerons ici comme exemple d'application celui des barrettes d'obturateurs pour têtes d'impression.

La demande actuelle pour les télécopieurs, les traitements de texte et les imprimantes pour ordinateurs portables a fait naitre le besoin d'imprimantes sans impact, compactes, légères et de faible coot. C'est pourquoi des imprimantes photo-électrographiques comportant des barrettes d'obturateurs à cristaux liquides (LCLSA:liquid crystal light shutter arrays) sont à l'étude depuis maintenant 4 ans.

Umeda et al. chez Hitachi $[27,28]$ ont démontré la faisabilité d'une ligne d'obturateurs multiplexés à cristaux liquides ferroélectriques utilisant une méthode de stabilisation en champ alternatif. La structure de leur tête d'impression est représentée sur la figure 3. La séquence de commutation et de stabilisation (Fig.4) utilise des impulsions de $20 \mathrm{~V}$ Cont., séparées par des séquences en alternatif (tension VH) à $45 \mathrm{~V}$ et $25 \mathrm{kHz}$, superposées à une rampe continue, ce qui a pour effet de réduire notablement le changement de transmission lumineuse (Fig. 5):quand VS est appliqué seule, la variation est grande, mais quand on applique VS+VH, la variation est de moins de 20 o de la transmission initiale. $L^{\prime}$ épaisseur de cristal liquide est de 4,4+0,4 um et la ligne d'obturateurs est au pas de 10 points/mm; le temps d'impression par ligne de 1,25 ms permet 1 'impression de 12 pages par minute.

Un tavail un peu semblable a été fait à NEC par Naemura et al. [29].

\section{MODULATBURS OPTIQUES}

Les propriétés de commutation des cristaux liquides ferroélectriques permettent la fabrication de modulateurs optiques spatiaux rapides et à haut contraste pour des applications telles que le traitement optique du signal (par exemple le traitement des images, qui demande de grandes vitesses de données) ou le calcul optique (par exemple portes logiques, crossbars optiques, masques de filtrage spatial). Un réseau linéaire de valves optiques a été réalisé dans une version préliminaire dès 1983 par Clark et al.et se trouve décrit, ainsi que les améliorations ultérieures dans la référence [21]. La version améliorée comportait 256 bandes, chacune de largeur $90 \mu \mathrm{m}$ espacées de $10 \mu \mathrm{m} ; 1^{\prime}$ épaisseur de cellule était de 1 um grace à des cales d'épaisseur en Sio de $30 \times 30 \mu \mathrm{m}$. La commutation entre les deux états stables (stabilisés par effet de surface) était obtenue par de brèves impulsions de durée 10 et $100 \mu \mathrm{s}$. Des temps de réponse inférieurs a $1 \mu s$ dans la phase SmC* ont été annoncés et certaines petites zones des cellules de test ont même pu être comnutées avec des impulsions d'environ $100 \mathrm{~ns}$ sous $40 \mathrm{~V}$. plusieurs matériaux cristaux liquides ont été utilisés, comme par exemple le MBRA et le HOBACPC, qui présentent une phase $\mathrm{SmC}^{\star}$ dans la gamme $40-65^{\circ} \mathrm{C}$. 
Plus tard, Armitage et al. [30] ont comparé les nématiques rapides et les ferroélectriques pour les modulateurs spatiaux de lumière (SLM:spatial light modulator). Ils ont proposé d'utiliser des ferroélectriques stabilisés par les surfaces (SSFLC) pour les modulateurs spatiaux dans les applications de taitement optique. Un banc de test de modulateur spatial a eté construit, qui comporte un SSFLC associé à une plaque de BSO (Bismuth Silicon oxide), insérés entre des substrats en verre (Fig.6 et 7). Pour des échantillons à alignement faible, ces auteurs ont pu observer non seulement la commutation à cadence vidéo, mais aussi le stockage d'informations pendant des durées significatives (plusieurs heures).

Nous avons signalé ci-dessus que la synthèse chimique appropriée et une gamme de température convenable permettent de contrôler l'angle $d^{\prime}$ inclinaison des molécules en phase $\mathrm{SmC}^{\star}$, par rapport à la normale aux plans smectiques. Lorsque le cristal liquide a été synthétisé pour être essentiellement optiquement uniaxe en phase SmC* et avoir une valeur de $22,5^{\circ}$ de $1^{\prime}$ angle $\mathrm{d}^{\prime}$ inclinaison sur une plage de température suffisamment large, alors, 1'axe optique, lié directement à la direction moléculaire, peut être tourné electriquement de $45^{\circ} ; \mathrm{si}$ de plus, 1'épaisseur de la cellule est convenablement choisie, on peut réaliser une lame demi-onde programmable électriquement. Ceci permet donc, par sélection électrique de l'état du ferroélectrique, soit de laisser inchangée une onde en incidence normale linéairement polarisée, soit de faire tourner sa polarisation de $90^{\circ}$.

L'utilisation de telles propriétés permet la réalisation sous forme matricielle de réseaux de portes optiques de faible pas. A Thomson-CSF, nous avons réalisé des cellules de test pour des dispositifs de $32 \times 32$ au pas de $250 \mu \mathrm{m}$ et pour des matrices de $256 \times 256$ au pas de $50 \mu \mathrm{m}$. La commutation optique est obtenue dès l'application d'une tension inférieure $\vec{a}+5$ ou -5 volts; le temps de commutation dépend de la tension appliquée; par exemple, sous une tension de + ou - $40 \mathrm{~V}$ à $2 \mathrm{kHz}$, un temps de réponse de $35 \mu \mathrm{s}$ a été observé à température ambiante.

De tels dispositifs peuvent être utilisés pour réaliser des réseaux de portes optiques pour le calcul optique et pour le traitement $d^{\prime}$ images [31-35] comme nous 1'avons signalé ci-dessus. Dans l'exemple décrit dans la référence [31], une matrice $32 \times 32$ a été réalisée, qui comporte des pixels de 17 um de côté espacés de $5 \mu \mathrm{m}$. Le contraste maximum mesuré à $100 \mathrm{~Hz}$ était de 1500:1 sur un point unique et de 125:1 sur le réseau matriciel. Des temps de commutation de $12 \mu \mathrm{s}$ a température ambiante et de $500 \mathrm{~ns}$ à plus haute température ont été annoncés dans cet article. Des portes logiques Booléennes de type XOR et XNOR utilisant les deux états de polarisation lumineuse ont été imaginées, avec l'avantage d'eviter la dissipation de puissance lumineuse et donc de permettre de placer de nombreuses portes optiques en cascade.

\section{CONCLUSIONS ET TENDANCES}

Les cristaux liquides chiraux ferroélectriques ont, comme les autres cristaux liquides, 1'intérêt de présenter de fortes valeurs de 1'anisotropie de 1'indice de réfraction et de pouvoir être commutés sous de faibles tensions de commande. Ils ont l'avantage supplémentaire de permettre un couplage linéaire en champ électrique, une commutation moléculaire très rapide (par rapport aux autres cristaux liquides), la bistabilité et un large angle de vue qui les ont déjà rendus très attractifs pour les applications en visualisation. L'élaboration de nouvelles molécules et la préparation de mélanges sont des étapes fondamentales vers des matériaux de basse viscosité, de faible temps de réponse et de grande stabilité à température ambiante. Les futures applications comporteront peut être des obturateurs pour des têtes d'impression compactes et de faible coût et des modulateurs optiques pour le traitement de signal et le calcul optique. Des dispositifs encore plus rapides (temps de réponse inférieur à $500 \mathrm{~ns}$ ) avec des pixels plus petits (taille inférieurs à $5 \mu \mathrm{m}$ ) et une meilleure qualité optique deviendront vraisemblablement très utiles pour les processeurs optiques parallèles à haut débit. 

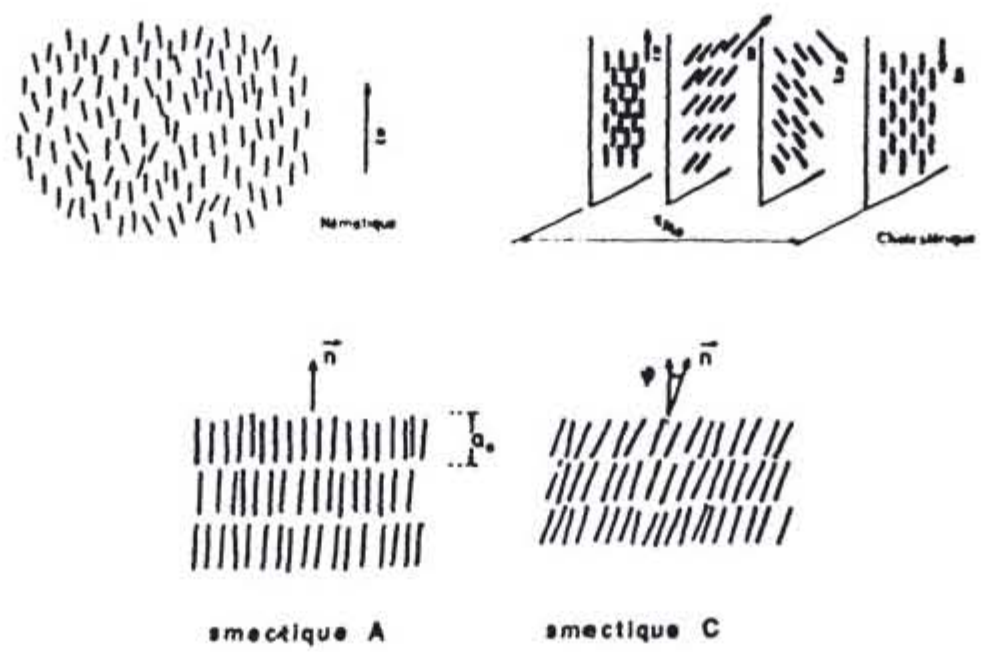

Arrangement des molécules dans les phases:

Nématique; Cholestérique; Smectiques A et C
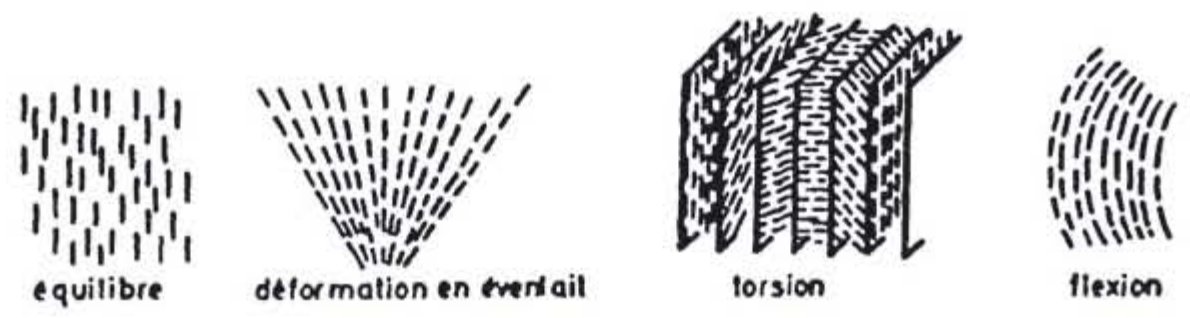

Les 3 modes de déformation

Divergence (éventail)

Torsion

plexion

F. aure 1: Symétries des cristaux liquides 


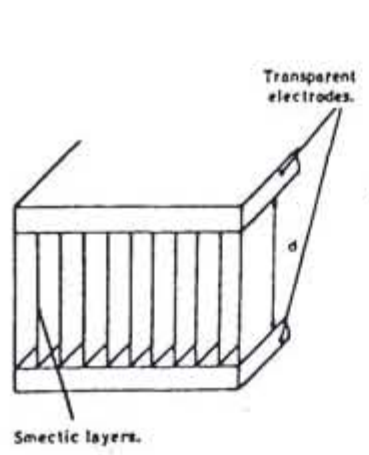

(a)

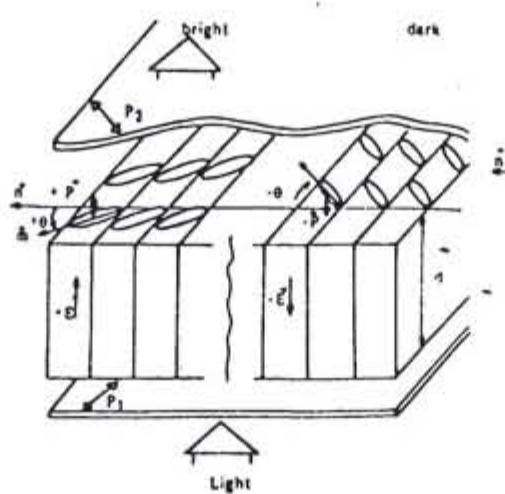

(b)

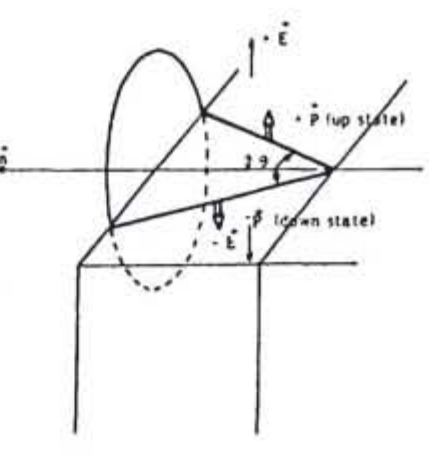

(c)

Figure 2

Surface Stabilized Ferroelectric Liquid Crystal Structure (SSFLC)

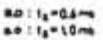

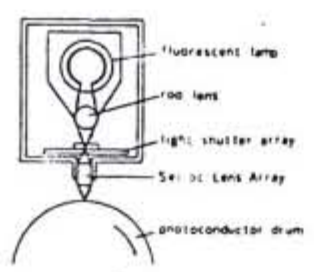
Fig.3 Stricture of aprint nex with
light hutile artog.

From Umeda et al. 227-28

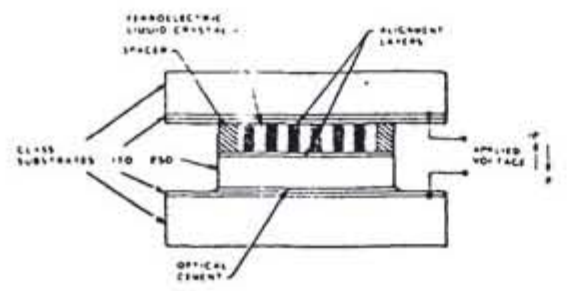

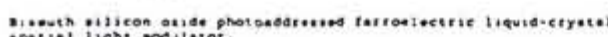
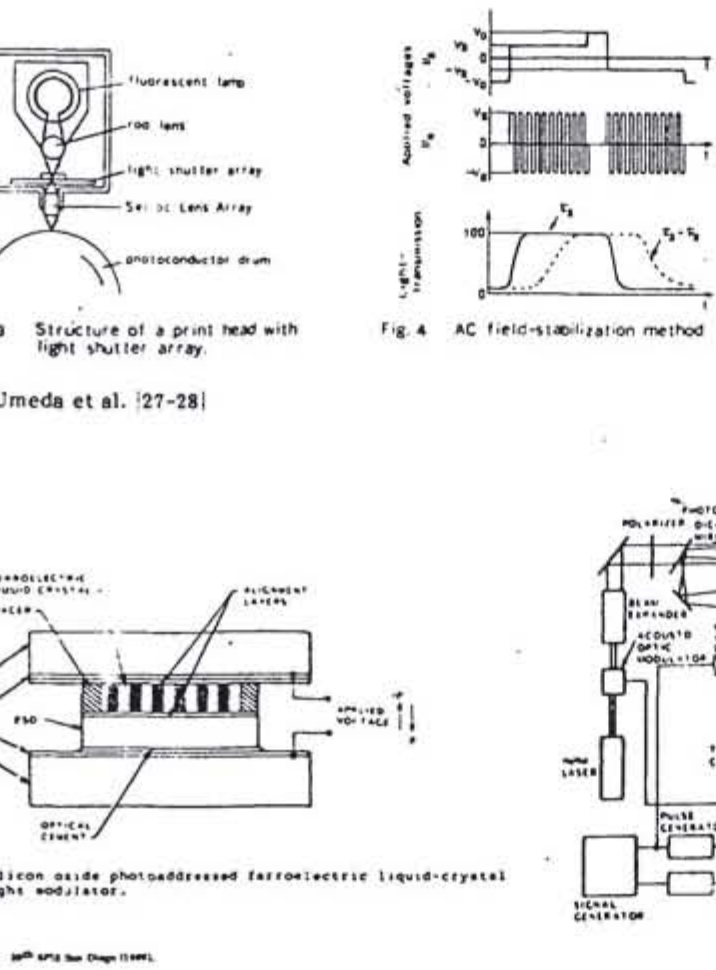

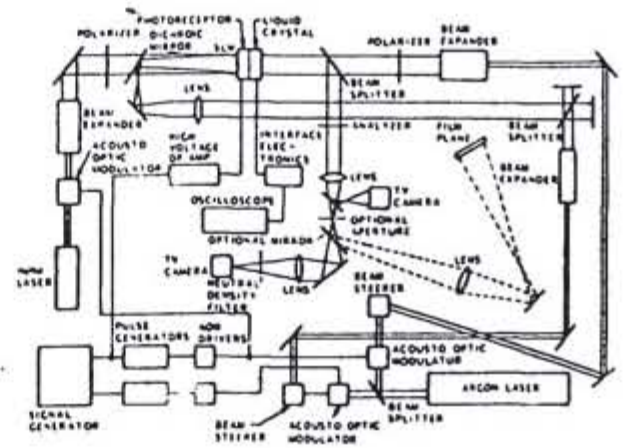

cooplete opellet itght nodoluter teet beo.

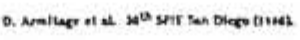

Figure 7

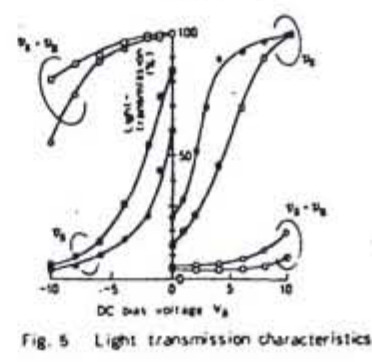

Fig. I Light tomsmission cherateristica 
CL. NEMUTQUES

COURLACE DU DRPOLE DNDUIT AVEC $\vec{E}$

$\Omega_{E}=\Delta E E^{2} \sin \varphi \cos \varphi^{\circ}$

$\Omega_{E}=$ COUPLE ELECTRQUE

Ae - द - E - ANISOTROPIE DE PERMTTINTIE

$\bar{E}$ - champ electruque apPLLqe - = orientation du directeur N̂ par

RORCES VISCOELASTOUES

$$
\Omega_{\mathrm{V}}=K \frac{d^{2} \hat{\mathrm{N}}}{d^{2}} \cdot \eta \frac{d \hat{\mathrm{N}}}{d t}
$$

K - Modure enastique

N - directeur pour lormatation MOLECILURE LOCNIE

z - COORDONNEE SPATULE

$\eta$ - viscosire

C L FERROELECTRUQUES

CQUPLAGE AVEC LA POLNUSADON FERROEECTRIOUE

$$
\Omega_{P}=P E S D N
$$

$\Omega_{p}:$ COUPLE

$\bar{p}$ : POLARISATION FERROELECTRIQUE

$\vec{E}$ : CHAMP ELECTRQUE NPPUQUEE

$\checkmark$ : NNGLe entre $\vec{P}$ Et $\vec{E}$

COUPLAGE AU DTPOLE NDOUIT

$$
\Omega_{E}=\Delta E^{2} \sin \zeta \cos \zeta
$$

$\Omega_{E}:$ COUPLE

AE : ANISOTROPIE DE PERMITIVITE

$\vec{E}$ : CHNP EIECTRIQUE

6 : NNGLE ENTRE N ET $\bar{E}$
C.L NEMATIQUES

TEMPS DE RERONSE (ORIENTATON PARALIELE)

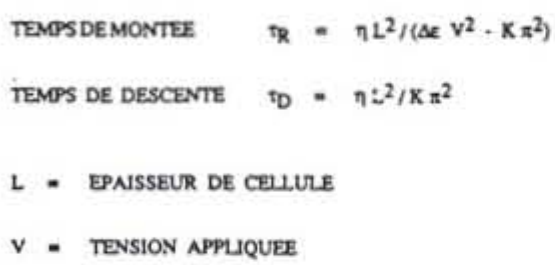


Les cristaux liquides ferroelectriques...
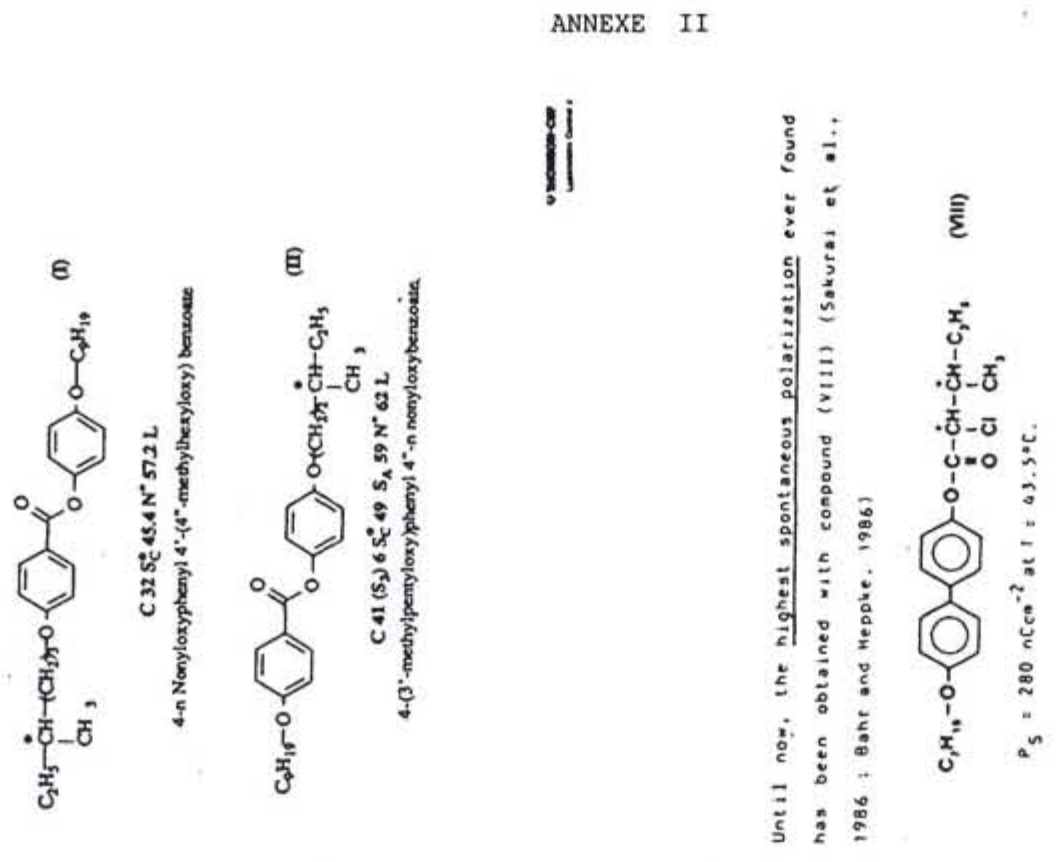

ij
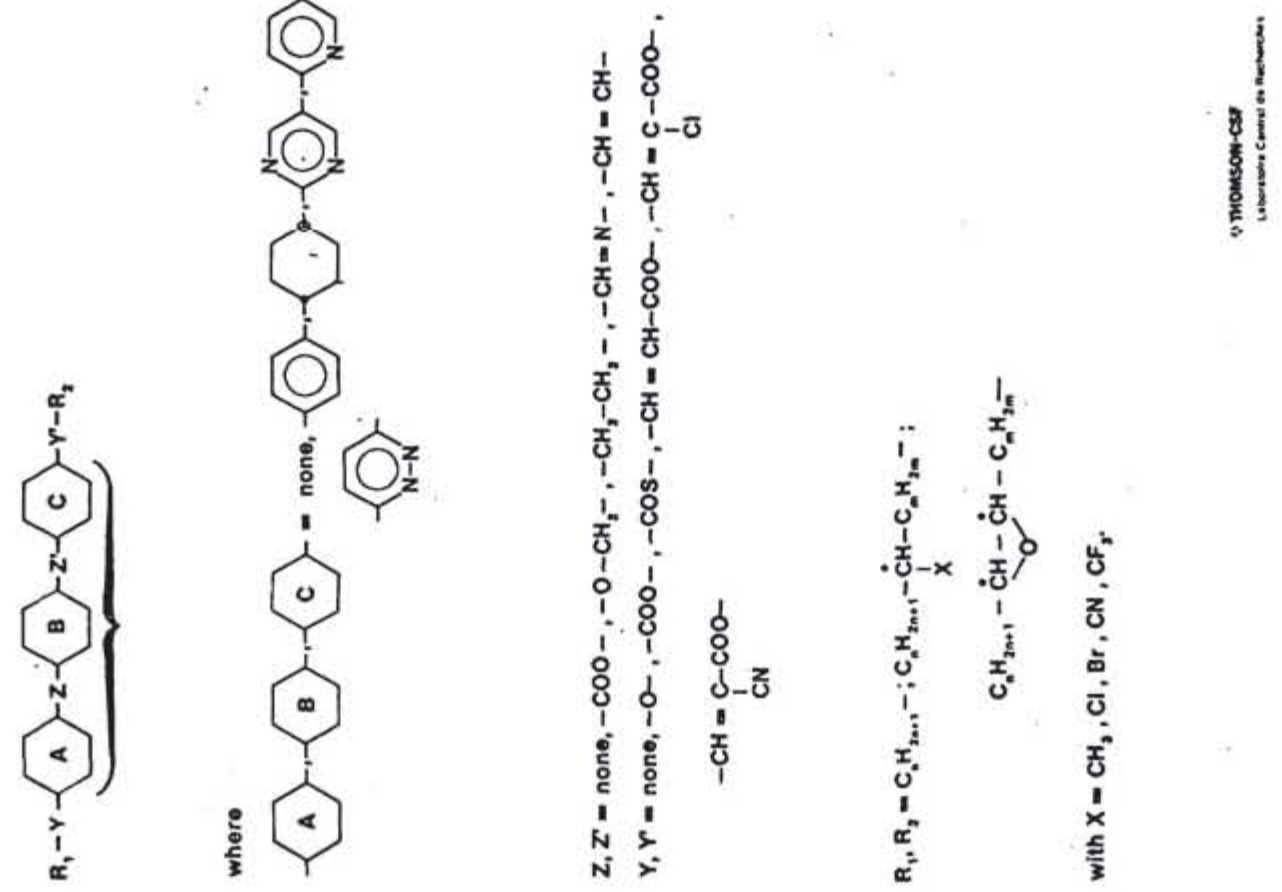
ANNEXE III

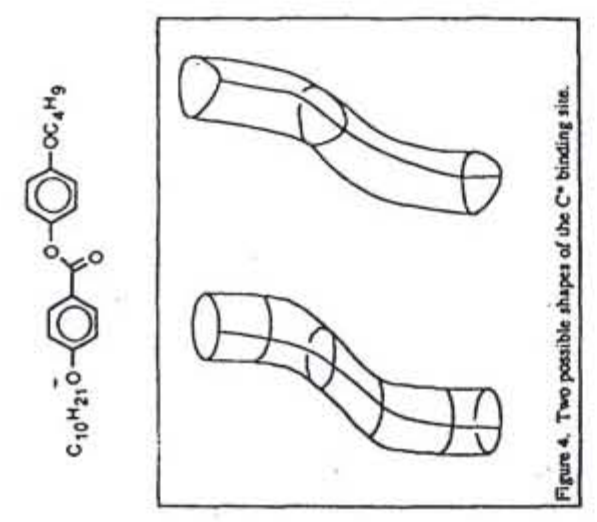

!ٕ!

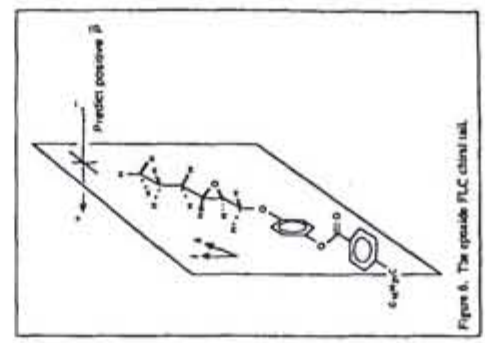

8
$\frac{1}{8}$
8
$\frac{8}{5}$
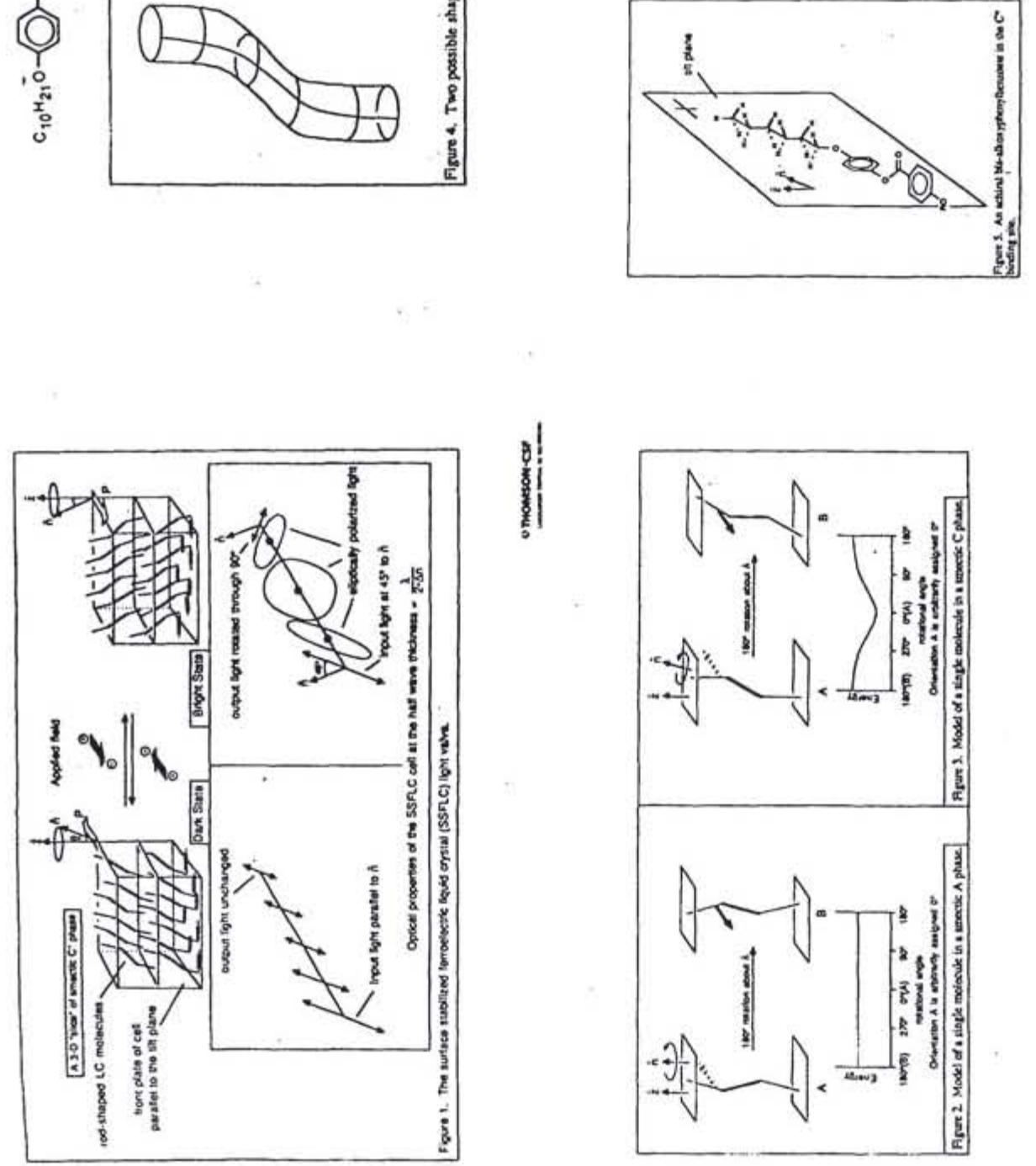

!े!

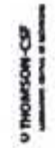

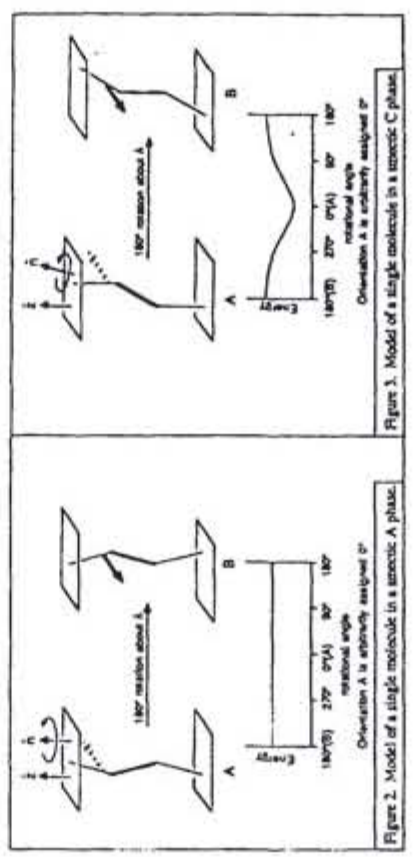




\section{REFEREVITES}

III P. Le Barny and J.C. Dubois, Side Chain. Liq. Cryst. Polym. C.B. Mc Ardle Edt. Chap. V to be published.

|2| J. Mlegarino - Appl. Phys. A, 11, 297 (1986) J.P. Le Pesant - Opto $8817-19$ May 1988 Paris ESt Publications.

|3| D.J. Grant, B.K. Jenes and s.G. Clark, Int. Disp. Res. Conf. "Eurodisplay 87" Cont, Proceed. p.67 (1987). [4] R.B. Meyer, L. Liebert, L. Strzelecki and P. Keller J. Phys. Paris Lett. 36, L 69 (1975).

|S| P. Martinot-Lagarde, R. Duke and C. Durand - Nol. Cryst. Liq. Cryst. 75, 249 (1981).

|6| T. Geethar 1 rst international Symposium on Ferroelectric Liquid Crystals Arcachon (1987).

|7| C. Destrade, J. Malthete, Nguyen Huu Tinh, Mol. Cryst. Liq. Cryst., 127, 273 (1985).

|8| J. Goodby, T. Leslie, Mol. Cryst. Liq. Cryst., 110, 175 (1984).

|9| B. Otterholm, M. Nilsson, S. Lagerwall, K. Skarp, Liq. Cryst., 2 (6), 757 (1987).

$|10|$ M. Bradshaw, J. Constant, $E$. Raynes, The British Liquid Crystal Society Annual conference, Alanchester (1988).

I11) B. Otterholm, C. Alstermark, K. Flatischler, A. Damlgren, S. Lagerwall, K. Skarp, Mol. Cryst. Liq. Cryst. 148,189 (1987).

|12| J. Patel, J. Goooby, Optical Engineering, 26, (5) 373 (1987).

$|13|$ N. Clark, S. Logerwall, Ferroelectrics 59, 25 (1984).

114| T. Sakurai, N. Mikami, R. Higuchi; M. Honme, M. Ozadi, K. Yoshino, J. Chem. Soc. Chem. Commun, 978 (1986).

(15 C. Bahr, G. Heppke, Mol. Cryst. Liq. Cryst. Letters, 4 (2), 31 (1985).

116; 1. Sage, J. Jenner, M. Chambers, M. Bradshaw, v. Brimmell, J. Constant, J. Hugues, E. Raynes, A. Samra, G. Gray, D. Lacey, K. Toyne, L. Chan, 1. Shenouda, A. Jackson $1^{\text {rst }}$ International Symposium on Ferroelectric Liquid Crystals Arcachon (1987).

|17| N.A. Clark, S.T. Logerwall. Appl. Phys. Lett. 36, 999, 1980.

S.T. Lagerwall, B. Otterholm, K. Sharp. Nol. Cryst. Liq Cryst. 152 (pt B), S0,-87, 1987.

(18| J.P. Le Pesant, J.N. Perbet, 8. Mourey, N. Hareng, G. Decobert and J.C. Dubois, 10th Inter. Liq. Cryst. Cont., York, G.B., 15-21 July 1984. Published in Mol. Cryst. Liq. Cryst. 129, 61 (1985).

[19! J.S. Patel, T.M1, Leslie, J.1v, Goobby, Ferroelectrics 59 , 137 (1984).

$|20|$ C. Bowry, A. Mosley, B.M. Nicholas, F. Le Texier, J.P. Boye:, J.P. Le Pesant, J.F. Clere J. Dijon and C. Ebel, 1 st Int. Symp. Ferto. Liq. Cryst., Arcachon, F. (1987), to be published in Ferroelectrics.

|21| N.A. Clark, S.T. Lagerwall and J. Wahl, Proceed. SID 2612, 133 (1985).

|22| J. Dijon. P. Maltese, C. Ebel, Proceed. Eurodisplay 1987, p. 40 (1987).

123: M. Brunet, N. Iseert, 1 s! Int. Symp. Ferra. Liq. Cryst., Arcachon, F (1987) to be published in Ferreelectrics.

$124 \mid$ W.A. Crossland, M. Bone. F.W. Ross - Proceed. Eurodispley 1987 p. 29 (1987).

|25| S. Matsumoto, A. Mura ama, H. Hatoh, Y. Kinoshita, H. Mirai, M. Ishikawa, S. Kamagami, SID 88 Digest, 41 (1988),

[26! J. Dijor, C. Ebel, C. Vauchier, F. Baume J.F. Clerc, M. Estor, T. Leroux, P. Maltese, L. Mulatier, SID 88 Digest, 246 (1988).

2: T. Umeda, Y. Hori and A. Mukoh, SID 85 Digest, 373 (1985).

|33| J.S. Patel Opt. Tng. 26. 129 (1987),

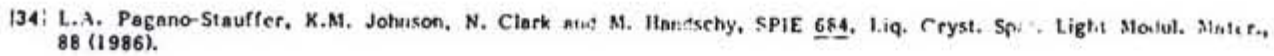

135: M.A. Handschy, K.1., Johison, W.T. Cathey and I. A. Pogano-Stauffer, Opt. 1.et1. 12, 611 (1983). 
i281 T. Umeda, T. Nagats, A. Nukoh and $Y_{-}$llori, S1D 87 Digest, 352 (1987).

$|29|$ S. Naemura, H. Ichinose, Y. Kato, H. Leda, A. Kogo, K. Ohsowa, S. Ishıznki, H. Kilayama and V. Yukino, SID 87 Digest. 356 (1987).

|30| D. Armitage. J.I. Thackara, N. Clark and Y.A. Hanc hy, 30 th SPIE, San Diego, L'SA (1985) see aiso : Mlolec, C:yst. Liq. Cryst. 144, 309 (1987).

|J1| K.M. Johnson, M.A. Handschy and L.A. Pagano-Stauffer, Opt. Engin. 26, 385 (1987).

[32] G. Moddel, K.M. Jolinson and N.A. Hlandsehy, SPIE 754, Opt. Dig. Patt. Recog., 207 (1987).

[36] D.M.Walba and N.A.Clark, SPIE Vol.825 Spat.Light Modul.Applic. II, 81 (1987).

[37) T.P.Rieker et al., Phys.Rev.Lett, Vol.59,2658 (1987). 